\title{
Diversity of intervention effects: Unifying three Mandarin Chinese dou constructions*
}

\author{
Ciyang Qing \\ Stanford University
}

\begin{abstract}
I propose a novel and principled way to combine Hamblin/KratzerShimoyama style question semantics and Rooth's focus semantics and show that it provides a unified semantic account of the seemingly idiosyncratic ordering restrictions in three Mandarin constructions with the preverbal focus-sensitive particle $d o u$, in terms of various intervention effects.
\end{abstract}

Keywords: focus sensitivity, question semantics, intervention effects, Mandarin Chinese particle dou

\section{Introduction}

This paper concerns three Mandarin Chinese constructions with the focus-sensitive particle dou. These constructions involve wh-questions/indeterminates and focus sensitivity, and have seemingly idiosyncratic ordering restrictions. Two research questions naturally arise from such data: (i) how do we combine focus and question semantics to capture the meanings of these constructions and derive them compositionally, (ii) how do we make sense of the ordering restrictions. I will argue that these two questions can receive a unified answer.

The rest of the paper is organized as follows. In Section 2, I introduce the three Mandarin Chinese dou constructions, including their meanings and ordering restrictions, i.e., constraints on the relative positions of $d o u$ and its focus associate, and suggest that the patterns can be viewed as intervention effects. In Section 3, I introduce a novel and principled way to combine question and focus semantics. In Section 4, I provide a formal analysis of the three dou constructions and illustrate how their ordering restrictions follow from interactions between the semantics of dou and other independently motivated semantic constraints. Further discussions and conclusion are in Section 5 and 6.

* I thank Daniel Lassiter, Cleo Condoravdi, Boris Harizanov for the great supervision throughout this project, and Beth Levin, Chris Potts, and other members of the Stanford syntax/semantics/pragmatics group for the valuable feedback. Many thanks to the reviewers and participants of CUSP 10, InqBnB2, SALT 28 for the very helpful comments and discussions.

C2018 Qing 


\section{Three Mandarin Chinese dou constructions}

\subsection{Exhaustive dou wh-questions}

First, dou can be used to exhaustify a wh-question.

Mandarin Chinese is a wh-in-situ, SVO language and uses a question intonation for interrogatives. A run-of-the-mill wh-question, such as John ate what? (1a), admits non-exhaustive/mention-some answers in appropriate contexts. ${ }^{1}$ For instance, in a context in which John went to a huge buffet last night, answering (1a) with lobster and crab is acceptable even if the answerer knows that John also ate other food. However, when the preverbal particle dou is used (1b), the question requires an exhaustive answer. In the same context as above, answering (1b) with lobster and $c r a b$ felicitously would require the answerer believe that John ate nothing else.
a. Yuehan chi le shenme ?
John eat PFV what ?
'What did John eat?'
wh-in-situ
b. Yuehan dou chi le shenme?
John DOU eat PFV what ?
'What all did John eat?'
exhaustive wh-question
c. * Shui dou chi le longxia? who DOU eat PFV lobster ?
Intended: 'Who all ate lobster?'
${ }^{*}$ wh $>$ dou
d. Dou (you) shui chi le longxia?
DOU (YOU) who eat PFV lobster ?
'Who all ate lobster?'
wh $<$ dou

In this construction, the wh-phrase must appear in a surface structural position that is lower than dou, e.g., when it is the object (1b). The construction is ungrammatical when the wh-phrase appears above dou, e.g., when it is the subject (1b). To express the intended exhaustive wh-question meaning, an existential construction using the auxiliary you 'to have' is used, where the preverbal particle dou appears above the wh-phrase (1d). ${ }^{2}$

1 For compactness, I will often use English glosses to refer to the original expressions in Chinese, and provide translations if the meanings are not obvious from the glosses.

2 For some speakers, the auxiliary you can be omitted in(1d), but the crucial point is that they also require dou be fronted and thus appear above the wh-phrase. 
Mandarin dou constructions

\subsection{Universal wh ... dou construction}

Second, dou can be associated with a focused wh-indeterminate (which is optionally marked with wulun) and the resulting construction has a universal reading (2a).

The wh-indeterminate must appear above dou, e.g., when it is the subject (2a). This construction is ungrammatical when the wh-indeterminate appears below dou, e.g., when it is the object (2b). In order to express the intended meaning of (2b), the object wh-indeterminate needs to be fronted to a position higher than dou, either sentence-initially (2c) or between the subject and $d o u(2 \mathrm{~d}) .^{3}$

a. [(wulun) shui $\left._{F}\right]$ dou piping le Yuehan . no.matter who DOU criticize PFV John .

'Everyone criticized John.' wh $>$ dou

b. *Yuehan dou piping le [(wulun) $\left.\operatorname{shui}_{F}\right]$.

John DOU criticize PFV no.matter who .

Intended: 'John criticized everyone.' $\quad{ }^{*}$ wh $<$ dou

c. [(wulun) $\left.\operatorname{shui}_{F}\right]$ Yuehan dou piping le _ .

no.matter who John DOU criticize PFV GAP .

'John criticized everyone' wh $>$ dou

d. Yuehan [(wulun) shui $\left._{F}\right]$ dou piping le _ .

John no.matter what DOU criticize PFV GAP .

'John criticized everyone' wh $>$ dou

So far we have seen that exhaustive $d o u$ wh-questions and universal wh ... dou construction have opposite ordering restrictions: the former requires that the whphrase appear below dou, whereas the latter requires that the wh-indeterminate appear above $d o u$.

\subsection{Scalar lian ... dou construction}

Third, dou can be associated with a focused constituent (optionally marked with lian) and the resulting construction has a scalar presupposition (3a).

The focused constituent must appear above dou, e.g., when it is the subject (3a). This construction is ungrammatical when the focused constituent appears below dou, e.g., when it is the object (3b). In order to express the intended meaning of (3b), the

3 Note that the most salient reading of some of the sentences might be different from the one specified here, particularly when wulun is absent. This is due to the structural ambiguity about whether the wh-indeterminate is the subject or the object. 
object needs to be fronted to a position higher than $d o u$, either sentence-initially (3c) or between the subject and $\mathrm{dou}(3 \mathrm{~d}) .^{4}$
a. [(lian) Mali $\left._{F}\right]$ dou piping le Yuehan
LIAN Mary DOU criticize PFV John
'Even Mary $F$ criticized John'
$\mathrm{XP}_{F}>$ dou
b. * Yuehan dou piping le [(lian) $\left.\mathrm{Mali}_{F}\right]$
John DOU criticize PFV LIAN Mary
Intended: 'John criticized even Mary $F$ '
$* \mathrm{XP}_{F}<$ dou
c. [(lian) $\left.\mathrm{Mali}_{F}\right]$ Yuehan dou piping le
LIAN Mary John DOU criticize PFV GAP
'John criticized even Mary $F$ '
$\mathrm{XP}_{F}>$ dou
d. Yuehan $\left[\left(\right.\right.$ lian) Mali $\left._{F}\right]$ dou piping le
John LIAN Mary DOU criticize PFV GAP
'John criticized even Mary $F$ '
$\mathrm{XP}_{F}>$ dou

\subsection{Interim summary}

The ordering restrictions in three Mandarin dou constructions are summarised below.

a. In exhaustive dou wh-questions, the wh-phrase associated with dou must appear below it.

b. In the universal wh ... dou construction, the wh-indeterminate associated with dou must appear above it.

c. In the scalar lian ... dou construction, the focused constituent associated with dou must appear above it.

Given that the three dou constructions discussed above involve questions and focus sensitivity, the following research questions naturally arise:

(5) a. How to represent the meanings of the three dou constructions and how to derive them compositionally? In particular, what is the contribution of dou?

b. How to explain the ordering restrictions in (4)?

I suggest that (5a) and (5b) are closely related and can be accounted for in a unified way. Anticipating the proposed analysis in the following sections, the ordering 


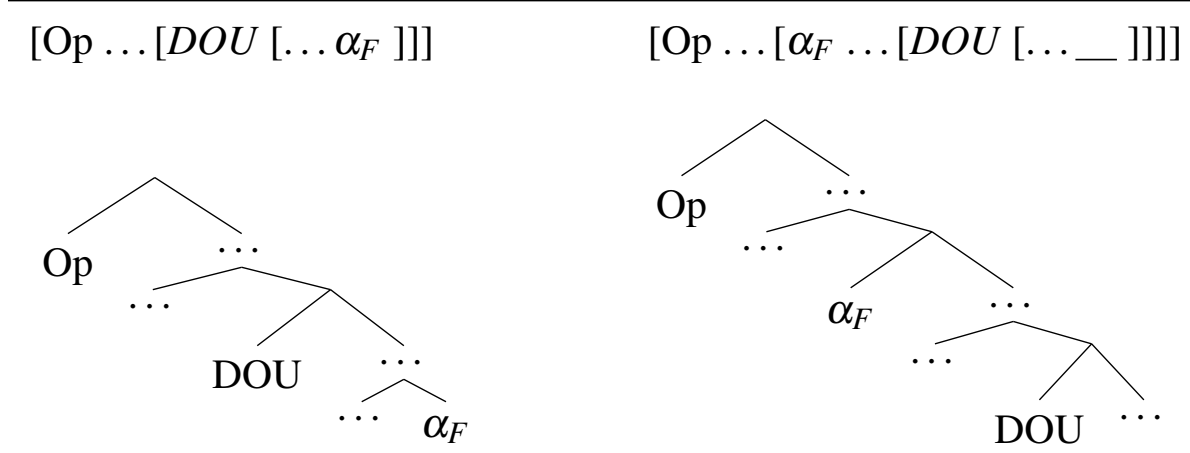

Figure 1 Two structural configurations for dou constructions: dou intervening between its focus associate $\alpha_{F}$ and a proposition-level operator Op (Left), or without such intervention (Right)

\begin{tabular}{ccl}
\hline Op & Focused constituent $\alpha_{F}$ & Effect of $\boldsymbol{D} \boldsymbol{O} \boldsymbol{U}$ intervening \\
\hline$[?]$ & wh $_{F}$ & wh-question exhaustification \\
{$[\forall]$} & (wulun) $\mathrm{wh}_{F}$ & $*$ \\
EVEN & (lian) $\mathrm{XP}_{F}$ & $*$ \\
Table 1 & Summary of the patterns as intervention effects \\
\hline
\end{tabular}

restrictions in (4) can be seen as a ban on one the two structural configurations in Fig. 1.

For each construction, we can think of its 'flavor' of meaning (i.e., question, universal quantification, or scalar presupposition, which will be made precise and formalized), as a proposition-level operator Op situated high up in the structure. The relative positions of $d o u$ and its focus associate $\alpha_{F}$ (a wh-phrase, wh-indeterminate, or a lian-XP) can then be equivalently characterized by whether dou intervenes between $\alpha_{F}$ and the high Op: if the focused constituent $\alpha_{F}$ appears below dou, it means that $d o u$ intervenes between $\alpha_{F}$ and Op (Fig. 1, left), and if the focused constituent $\alpha_{F}$ appears above dou then dou does not intervene between $\alpha_{F}$ and Op (Fig. 1, right). As a result, the patterns of the three dou constructions can be reformulated in terms of the effect of dou intervening between Op and $\alpha_{F}$, as summarised in Table 1. In the following sections, I will provide the semantics of the three dou constructions and derive these intervention effects.

4 Again, the most salient reading of some of the sentences might be different from the one specified here, particularly when lian is absent, due to the structural ambiguity about whether the focused constituent is the subject or the object. 


\section{Combining question and focus semantics}

Since the three dou constructions involve questions and focus sensitivity, providing their meaning representations requires (at least) combining question and focus semantics. The basic ingredients that I will use for this purpose are very familiar: a Hamblin-Kratzer-Shimoyama style semantics for questions and wh-indeterminates (Hamblin 1973; Kratzer \& Shimoyama 2002), and Rooth's two-dimensional focus semantics (Rooth 1985, 1992). However, I will combine the two ingredients in a novel way, resulting in a two-dimensional Roothian extension of Hamblin semantics.

This system has two main features: (i) the denotation of an expression is a set of ordinary-alternative value pairs, and (ii) inquisitiveness resides in ordinary values, i.e., ordinary values are Hamblin/Kratzer-Shimoyama style sets. ${ }^{5}$ Despite these differences from Rooth's original system, his main insight is maintained: the alternative value of an unfocused expression is the singleton set containing the ordinary value, whereas the alternative value of a focused expression is a set of contextually relevant ordinary values.

Let us consider a run-of-the-mill object wh-in-situ question John criticized $w_{h} o_{F}$ ? as a concrete example. The denotation of criticized has only one ordinaryalternative value pair (6a). The ordinary value in that pair is a singleton set $\{\mathbf{c r i t}\}$, since the expression is not inquisitive. And since the expression is not focused, the alternative value in the pair is a singleton set containing the ordinary value, i.e., $\{\{$ crit $\}$. The denotation of the F-marked wh-word who $F$ also contains only one ordinary-alternative value pair (6b). However, since who is inquisitive, the ordinary value of the pair is the set of (relevant) humans. And since the word is F-marked, its alternative value is a set of relevant ordinary values, i.e., singleton sets of humans (e.g., $\{\mathbf{m}\}$, which is $\llbracket$ Mary $\rrbracket^{o}$ ).

To make the denotations more easily readable, I will use tabular representations such as those in $(6 c)$.

$$
\begin{aligned}
& \text { a. } \llbracket \text { criticized } \rrbracket=\{\langle\{\text { crit }\},\{\{\text { crit }\}\}\rangle\} \\
& \text { b. } \llbracket \text { who }_{F} \rrbracket=\left\{\left\langle\left\{y \mid y \in D_{\text {human }}\right\},\left\{\{y\} \mid y \in D_{\text {human }}\right\}\right\rangle\right\} \\
& \text { c. } \begin{array}{rc} 
& \text { criticized } \\
\llbracket \cdot \rrbracket^{o} & \{\text { crit }\} \\
\llbracket \cdot \rrbracket^{a} & \{\{\text { crit }\}
\end{array} \\
& \begin{array}{cc} 
& \text { who }_{F} \\
\hline \llbracket \cdot \rrbracket^{o} & \{\mathbf{m}, \mathbf{b}, \mathbf{j} \ldots\} \\
\llbracket \cdot \rrbracket^{a} & \{\{\mathbf{m}\},\{\mathbf{b}\},\{\mathbf{j}\} \ldots\}
\end{array}
\end{aligned}
$$

5 At this point it might be unclear why the denotation of an expression is a set of ordinary-alternative value pairs rather than simply just one ordinary-alternative value pair. This is needed to allow the semantics of dou to create parallelism, which will become clear in the next section. The denotations of the expressions in this section are all singleton sets containing one ordinary-alternative value pair. For any such expression I will refer to the ordinary/alternative value of its only pair simply as its ordinary/alternative value. 
Mandarin dou constructions

Semantic composition in this new system is functional application or recursive pointwise composition, whichever is applicable. The formal recursive definition is as follows. ${ }^{6}$

(7) The result of composing $m$ and $n$, i.e., $m \circ n$, is

a. $m(n)$ or $n(m)$ if one can take the other as argument

b. $\left\langle m^{o} \circ n^{o}, m^{a} \circ n^{a}\right\rangle$ if both $m$ and $n$ are pairs

c. $\{x \circ y \mid x \in m, y \in n\}$ if both $m$ and $n$ are sets

Using the above definitions, the derivation of the sentence radical John criticized who $F$ is as follows (8).

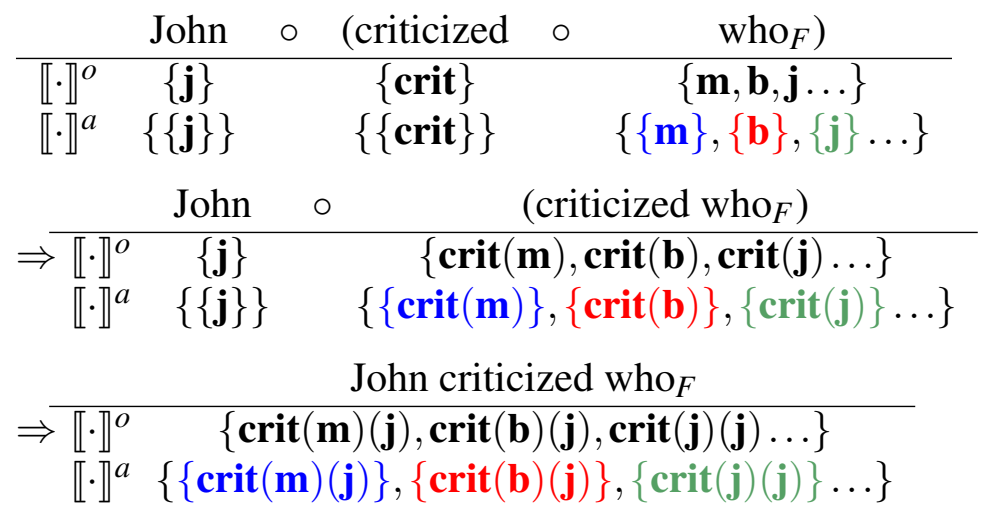

Finally, the interrogative force $\{[?]\}$ is applied to each ordinary-alternative value pair to compute its discourse effect. The definition of [?] is inspired by Roelofsen $\&$ Farkas (2015) and it ensures a proper Hamblin-style question denotation in the ordinary value, whose discourse effect is to request that the listener commit to at least one proposition in it (9).

$$
\begin{aligned}
& {[?](\langle m, n\rangle)=\left\langle m^{\prime}, n\right\rangle, \text { where } m^{\prime}=\{p, \neg p\} \text { if } m=\{p\} \text { is a singleton, otherwise }} \\
& m^{\prime}=m
\end{aligned}
$$

As a result, after applying the interrogative force, John criticized who? has the same denotation as in (8), since its ordinary value is not a singleton. Its discourse effect is to request the listener to commit to at least one proposition in the set $\{\operatorname{crit}(\mathbf{m})(\mathbf{j}), \boldsymbol{c r i t}(\mathbf{b})(\mathbf{j}), \boldsymbol{c r i t}(\mathbf{j})(\mathbf{j}) \ldots\}$. Therefore it allows for non-exhaustive answers.

The denotations of expressions in this section all contain only one ordinaryalternative value pair and therefore the discourse effects straightforwardly come from

6 Note that this compositional system is essentially stacking Charlow's (2014) focus and set monads and is fully principled. 
that one pair. When the denotation of an expression has multiple ordinary-alternative value pairs, its discourse effect is defined to be the cumulative effect of all those pairs. In the next section, I will illustrate how such cases are crucial in the derivations of the three Mandarin dou constructions.

\section{Deriving three Mandarin dou constructions}

\subsection{Exhaustive dou wh-questions}

To derive the denotation of an exhaustive dou wh-question, I propose that $d o u$ distributes the alternative values of its argument to create many ordinary-alternative pairs (10).

$$
\llbracket \text { DOU } \phi \rrbracket=\bigcup_{\langle m, n\rangle \in \llbracket \phi \rrbracket}\{\langle m \cap y,\{y\}\rangle \mid y \in n\}
$$

Let us break down the definition and see the intuition behind it. First, we can focus on cases in which the denotation of $\phi$ contains only one pair $\langle m, n\rangle$ : the same procedure is applied to each pair and the results are collected via set union. Second, for each $y$ in the alternative value of $\phi$ (i.e., for each $y \in n$ ), dou creates a new pair whose alternative value is a singleton set containing only $y$. The intuition is the following: if we think of the alternative dimension as the relevant domain of ordinary values, then what dou does is divide up the current domain into a set of smallest/atomic domains that only contain one element. These atomic domains are subsequently treated in parallel. Finally, the ordinary dimension can be viewed as a non-deterministic value in the domain specified by the alternative dimension. Therefore, as dou divides up the alternative dimension into smaller domains, the ordinary dimension needs to be restricted correspondingly, and hence the set intersection $m \cap y$ in the definition.

Given the above definition, we can derive the exhaustive dou wh-question (11).

Yuehan dou piping le $\operatorname{shui}_{F}$ ?

John DOU criticize PFV who ?

'Who all did John criticize?'

exhaustive wh-question

The full derivation is shown in Fig. 2. Given our composition rule, the many pairs created by composing dou with criticized who in Step 1 enter the remaining composition in a parallel fashion to get their discourse effects computed (Step 2-4). In the final step of the derivation, the question operator [?] takes each ordinary value and creates a set that corresponds to a yes-no question (Step 4). As a result, the cumulative discourse effect of (11) is "for each $x$, the speaker asks the listener whether John criticized $x$." The listener's answer is thus required to simultaneously address all these yes-no questions, i.e., it must be exhaustive. 


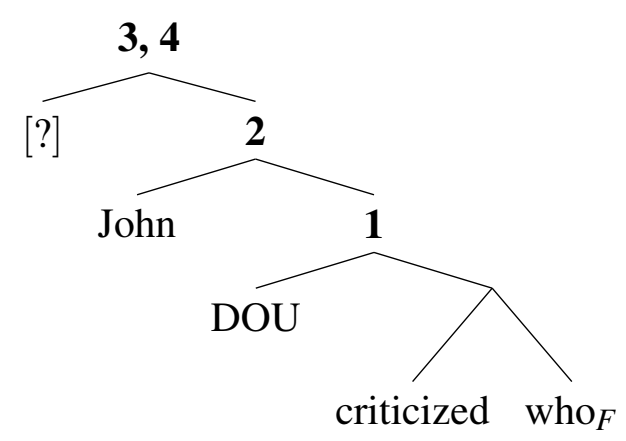

criticized who $F$

$\begin{array}{cc}\llbracket \cdot \rrbracket^{o} & \{\operatorname{crit}(\mathbf{m}), \mathbf{c r i t}(\mathbf{b}), \mathbf{c r i t}(\mathbf{j}) \ldots\} \\ \llbracket \cdot \rrbracket^{a} & \{\{\operatorname{crit}(\mathbf{m})\},\{\operatorname{crit}(\mathbf{b})\},\{\operatorname{crit}(\mathbf{j})\} \ldots\}\end{array}$

DOU criticized who $F$

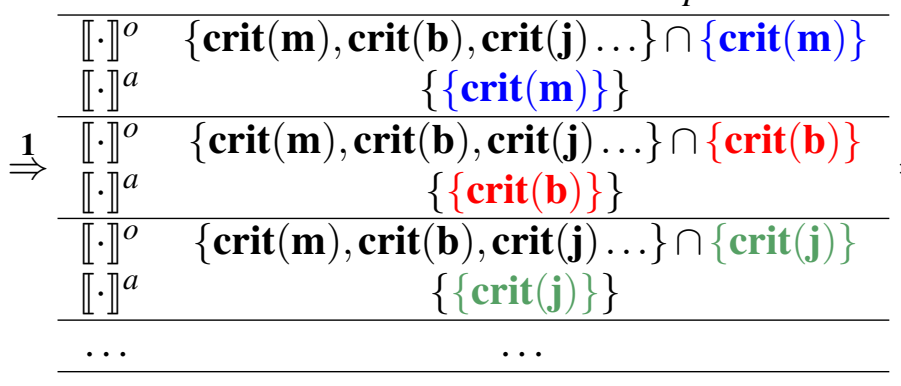

John DOU criticized who $F$

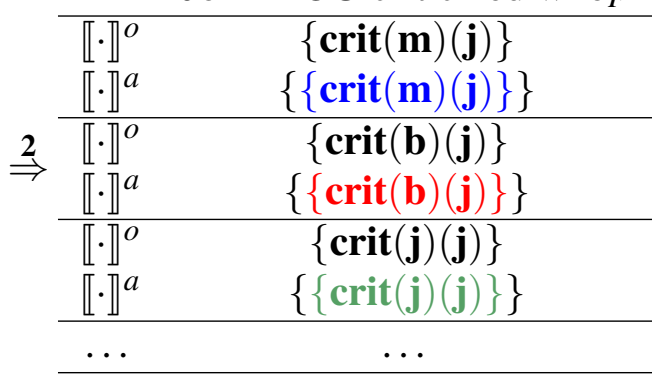

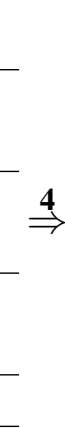

Figure 2 Derivation of the exhaustive dou wh-question John DOU criticized who $o_{F}$ ? 'who all did John criticize?' 
Since $d o u$, like many other Chinese expressions, only takes surface scope, such exhaustification can be achieved only when the wh-phrase is under the scope of dou. Therefore, in exhaustive dou wh-questions the wh-phrase must appear below dou.

We have seen that the proposed semantics of dou correctly predicts that it exhaustifies a wh-question when it intervenes between a wh-phrase and the [?] operator. Next, I will show that the semantics of dou in (10) and independent semantic constraints in the other two constructions interact to derive the remaining ordering restrictions.

\subsection{Universal wh ... dou construction}

For the wh ... dou construction, I adapt Rawlins's (2013) analysis of English unconditionals and assume a universal closure operator $[\forall]$ at the propositional level. $[\forall]$ is licensed by dou and licenses the optional wulun (assumed to be semantically vacuous $)^{7}$

Crucially, $[\forall]$ has an anti-singleton requirement in its definition (12a), which is independently motivated by (12b): the construction is ungrammatical if the ordinary value of the wulun-phrase is a singleton Mali he Su 'Mary and Sue', whereas it is fine with Mali haishi Su 'Mary or Sue', whose ordinary value is not a singleton, as can be shown by its ability to form alternative questions (12c).

a. $[\forall](\langle m, n\rangle)=\langle\{\bigwedge m\}, n\rangle$ if $m$ is not a singleton, otherwise undefined

b. Wulun Mali haishi/*he Su Yuehan dou piping le _ _ no.matter Mary or/and Sue John DOU criticize PFV GAP.

'John criticized Mary and Sue.'

c. Yuehan piping le Mali haishi Su ?

John criticize PFV Mary or Sue ?

'Did John criticize Mary or (did he criticize) Sue?'

When the focused wh-indeterminate associated with dou appears above it, e.g., when it is the subject (13), the derivation is shown in Fig 3.

[(wulun) $\left.\operatorname{shui}_{F}\right]$ dou piping le Yuehan . no.matter who DOU criticize PFV John .

'Everyone criticized John.'

$\mathrm{wh}_{F}>$ dou

Since the complement of dou (criticized John) is not focused and hence has a singleton set as its alternative value, composing it with dou does not change the denotation (Step 1). After the whole sentence has been composed (Step 3), the

7 This ensures that $d o u$ is obligatory and wulun only occurs in this construction. 

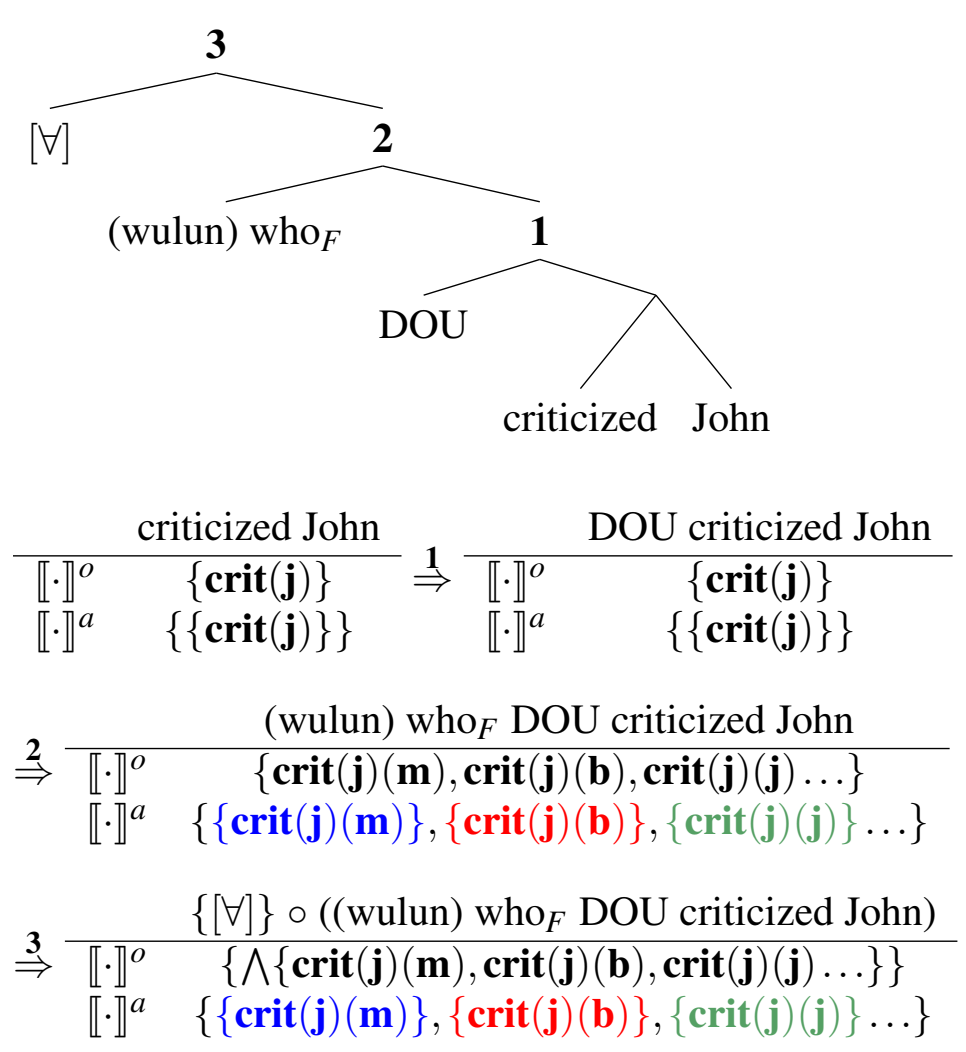

Figure 3 Derivation of the universal wh ... dou construction (wulun) who DOU criticized John. 'Everyone criticized John.'

informative content asserted is $\operatorname{crit}(\mathbf{m})(\mathbf{j}) \wedge \operatorname{crit}(\mathbf{b})(\mathbf{j}) \wedge \ldots$, which is the desired universal reading.

In contrast, if the focused wh-indeterminate associated with dou appears below it, e.g., when it is the object (14), the derivation is shown in Fig. 4.

* Yuehan dou piping le [(wulun) $\left.\operatorname{shui}_{F}\right]$.

John DOU criticize PFV no.matter who .

Intended: 'John criticized everyone.' $\quad *_{w h}<$ dou

Note that we have the same derivation as in the case of exhaustive dou whquestions up to the propositional level (Step 2) but will then apply universal closure $[\forall]$ rather than [?] (Step 3). Note that in this case the ordinary value in each pair is a singleton set, which violates the anti-singleton requirement of $[\forall]$. This correctly predicts that the wh-indeterminate cannot appear below dou in the universal wh ...dou construction. 


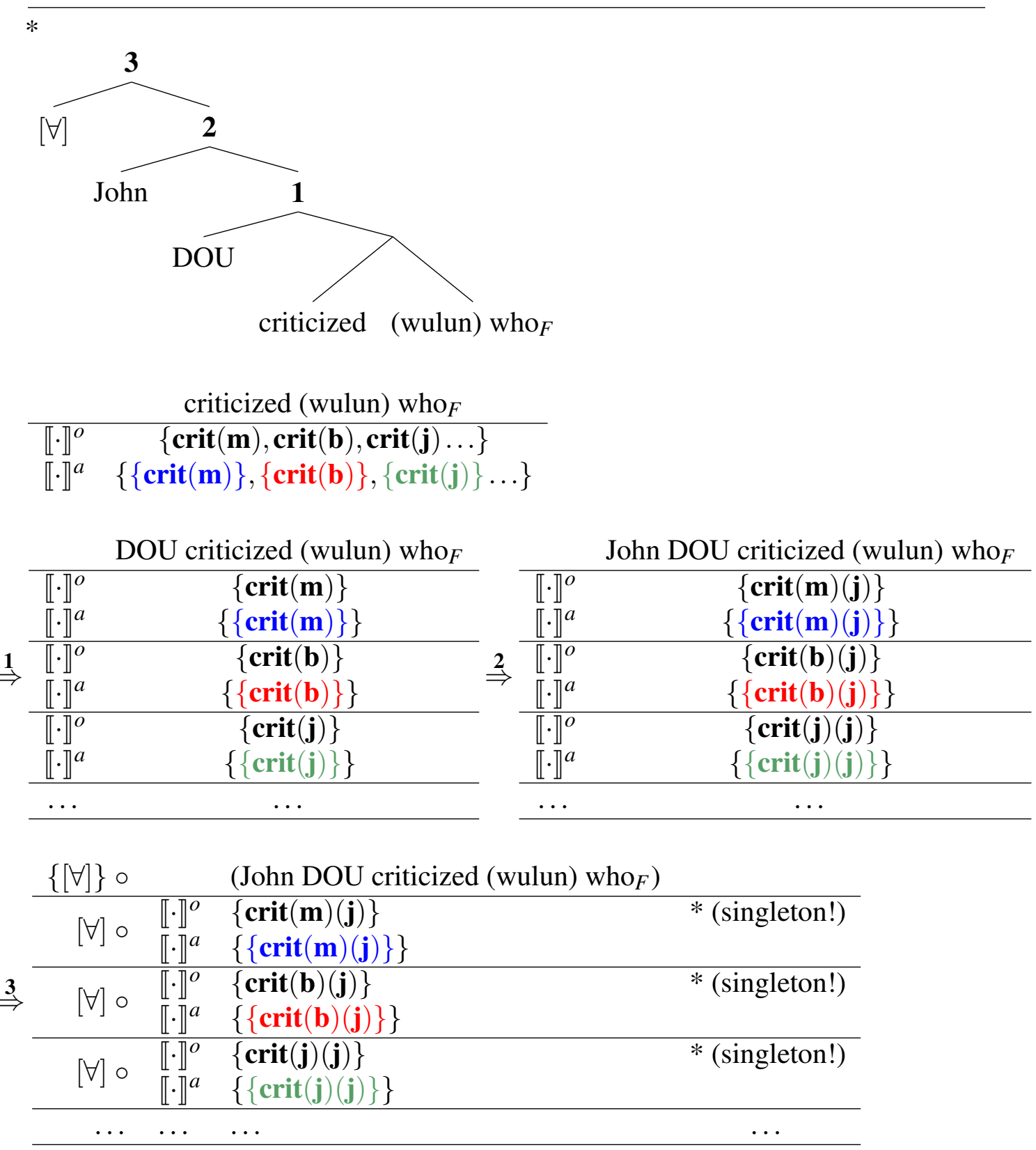

Figure 4 Derivation of the ordering constraint for universal wh ...dou construction, *John DOU criticized (wulun) who $F$ (intended: 'John criticized everyone'). 


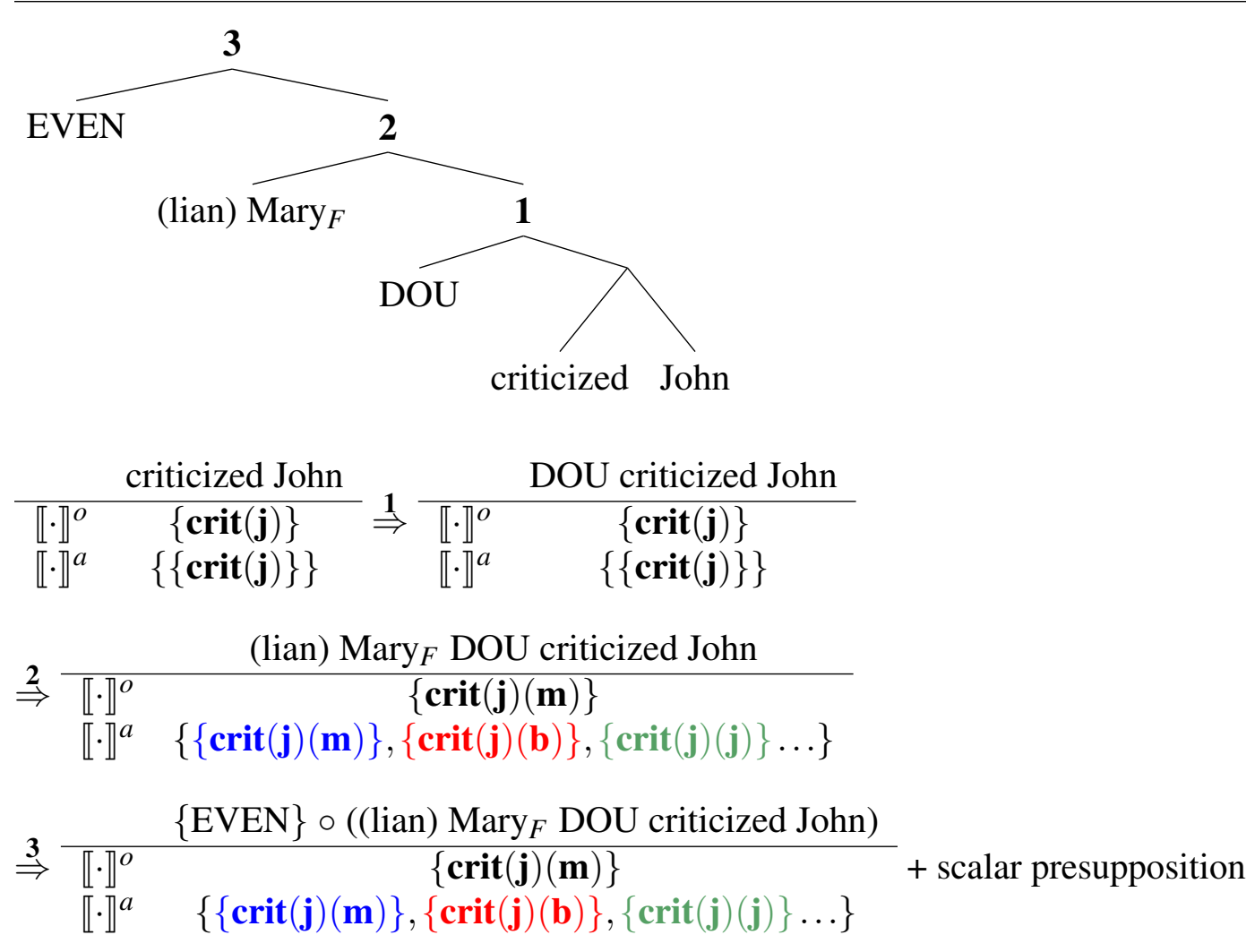

Figure 5 Derivation of the scalar lian ...dou construction (lian) Mary ${ }_{F}$ DOU criticized John 'Even Mary $F$ criticized John.'

\subsection{Scalar lian ... dou construction}

Finally, for the lian ...dou construction, similar to the analysis of the wh ...dou construction, I assume a scalar operator EVEN at the propositional level, which is licensed by dou and licenses the optional lian (assumed to be semantically vacuous).

(15) $\operatorname{EVEN}(\langle m, n\rangle)=\langle m, n\rangle$, with the scalar presupposition that $m$ is unlikely/the least likely among alternatives in $n$.

When the focused constituent associated with dou appears above it, e.g., when it is the subject (16), the derivation is shown in Fig 5.

$$
\begin{aligned}
& \text { [(lian) } \left.\text { Mali }_{F}\right] \text { dou piping le Yuehan } \\
& \text { LIAN Mary DOU criticize PFV John } \\
& \text { 'Even Mary } F \text { criticized John' } \\
& \mathrm{XP}_{F}>\text { dou }
\end{aligned}
$$

Again, since the complement of $d o u$ is not focused and hence has a singleton set as its alternative value, composing it with dou does not change the denotation 


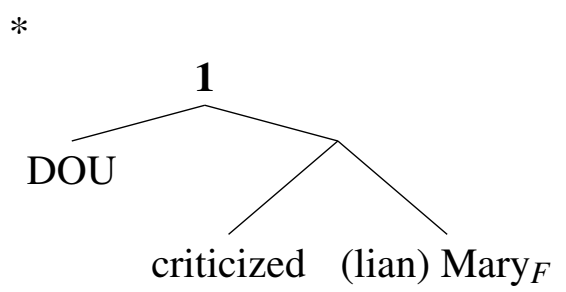

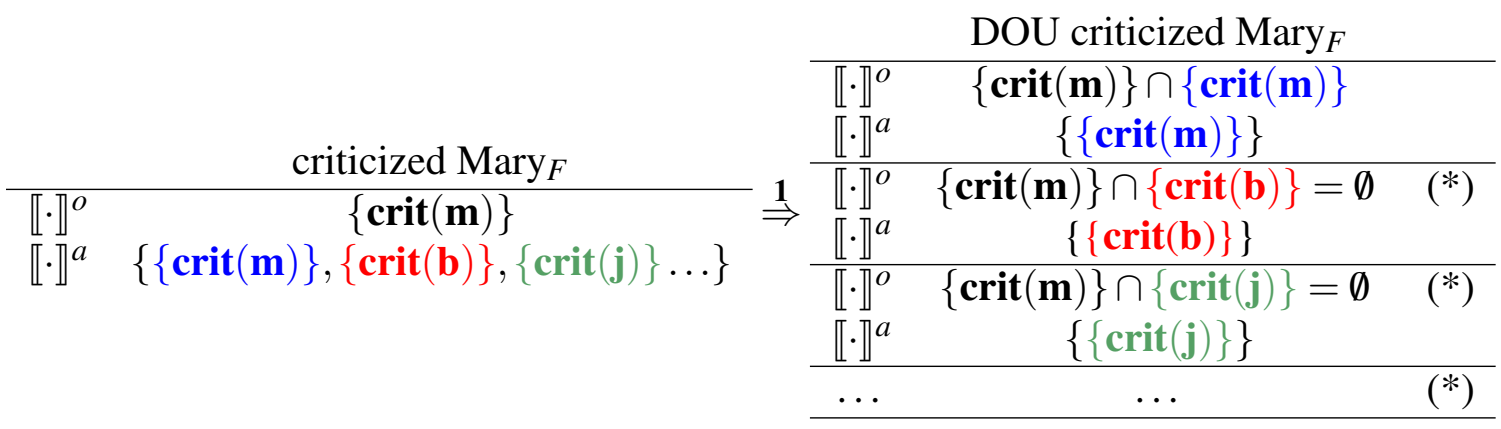

Figure 6 Derivation of the ordering constraint of the scalar lian ...dou construction: *John DOU criticized (lian) Mary $F_{F}$ (intended: 'John criticized even $\operatorname{Mary}_{F}$. ')

(Step 1). After the whole sentence has been composed (Step 3), the informative content asserted is that Mary criticized John. In addition, EVEN introduces the scalar presupposition that Mary criticizing John is unlikely/the least likely among alternatives such as Bob criticizing John, John criticizing himself etc. This is the desired scalar reading.

In contrast, if the focused constituent associated with dou appears below it, e.g., when it is the object, the derivation is shown in Fig. 6.

$$
\begin{aligned}
& \text { * Yuehan dou piping le [(lian) } \left.\mathrm{Mali}_{F}\right] \\
& \text { John DOU criticize PFV LIAN Mary } \\
& \text { Intended: 'John criticized even Mary } F_{F}
\end{aligned}
$$

Crucially, composing the lian-focus and dou results in empty sets in some of the ordinary values, crashing further composition because ordinary values are required by definition to be non-empty sets. This correctly predicts that the lian-focus cannot appear after dou in the scalar lian ... dou construction. 
Mandarin dou constructions

\begin{tabular}{ccl}
\hline Op & Focused constituent $\alpha_{F}$ & Effect of $\boldsymbol{D} \boldsymbol{O} \boldsymbol{U}$ intervening \\
\hline$[?]$ & $\mathrm{wh}_{F}$ & wh-question exhaustification \\
{$[\forall]$} & (wulun) $\mathrm{wh}_{F}$ & anti-singleton requirement violation $(*)$ \\
EVEN & (lian) $\mathrm{XP}_{F}$ & $\llbracket \cdot \rrbracket^{o}=\emptyset$, crash in composition
\end{tabular}

Table 2 Summary of the analysis

\subsection{Summary}

Now let us summarise how the proposed analysis addresses the research questions in (5), repeated below in (18).

(18) a. How to represent the meanings of the three dou constructions and how to derive them compositionally? In particular, what is the contribution of dou?

b. How to explain the ordering restrictions in (4)?

For (18a), I proposed a Roothian extension to the Hamblin/Kratzer-Shimoyama style question semantics to incorporate both questions and focus sensitivity. The meaning representations are layered with three notions of alternatives involved (19).

a. Non-determinism: The inner layer, i.e., the ordinary dimension $\llbracket \cdot \rrbracket^{o}$, is a set that represents a (potentially) non-deterministic value

b. Domain alternatives: The next layer, i.e., the ordinary-alternative value pair, encodes the information of alternative values in the domain in the alternative dimension $\llbracket \cdot \rrbracket^{a}$

c. Parallelism/family of propositions: The outer layer, i.e., a set of ordinaryalternative value pairs, represents the parallel treatment of of each of its elements and the overall discourse effect is calculated cumulatively

Despite the surface complexity, each of these notions is very familiar in the literature. Moreover, the system is modular: each layer is essentially a type constructor/transformer, i.e., a recipe that allows us to build even more complex systems in a principled way. For instance, if for some reason we decide that the Hamblin/KratzerShimoyama style semantics in the inner layer is inadequate and we want to instead use, e.g., compositional inquisitive semantics (Ciardelli, Roelofsen \& Theiler 2017), we can still apply the recipes in (19b) and (19c) to straightforwardly build a new system without affecting the core predictions.

As for the semantic contribution of $d o u$, I proposed that it distributes the alternative values of its argument and creates multiple ordinary-alternative value pairs that 
enter further semantic composition in parallel. The exhaustive question reading can then be captured as the cumulative discourse effect of a family of polar questions.

Finally, for (18b), I showed that the ordering restrictions can be seen as effects of the particle dou intervening between its focus associate $\alpha_{F}$ and a higher operator Op at the propositional level, and can be derived from the interaction between the semantics of dou and other independently motivated semantic constraints. The analysis is summarised in Table 2.

\section{Discussion}

\subsection{Comparison with previous analyses of $d o u$}

Despite the vast literature on dou, previous analyses (e.g., Shyu 1995; Cheng 1995; Lin 1996, 1997; Giannakidou \& Cheng 2006; Xiang 2008; Liao 2011; Tsai 2015; Liu 2017) tend to overlook or exclude the use of dou in exhaustive wh-questions and treat the ordering restrictions as syntactic idiosyncrasies. The current analysis, to my knowledge, provides the first semantic explanation of these ordering restrictions, which are derived from the interaction between the semantics of dou and other independently motivated semantic constraints.

The main challenge a purely syntactic analysis faces is that the ordering restrictions are not uniform: the focus associate $\alpha_{F}$ of dou must appear below it in exhaustive wh-questions and above it in the other two constructions. A representative syntactic approach (e.g., Shyu 1995) treats F-marking on the focus associate $\alpha_{F}$ as the trigger of a focus-movement to the specifier of $d o u$ (and may be moved up further). Now, in order to capture the fact that $\alpha_{F}$ must be higher than $d o u$ in the universal wh ...dou and scalar lian ...dou constructions, a purely syntactic account would need to require this movement be obligatory. However, given that the wh-phrase associated with dou in an exhaustive wh-question need not (and in fact, cannot) move up to a position higher than dou, one would need to assume either (i) the particle dou in exhaustive questions is a different one from the rest of the constructions, which is an unattractive last resort, or (ii) the wh-phrase in an exhaustive wh-question does not in fact bear F-marking and therefore does not undergo the same movement in other constructions. However, a consequence of (ii) is that interpretative mechanism of focus sensitivity is non-uniform: in addition to the standard mechanism through which a focus-sensitive operator access its quantificational domain via the non-singleton alternative dimension generated by F-marking, one would need to allow for the possibility for a focus-sensitive operator to get its quantificational domain elsewhere when its associate is not F-marked. For instance, Li \& Law (2016) propose that a focus-sensitive operator can use the ordinary value of a non-F-marked wh-phrase as its quantificational domain, and use 
this as the basis of their analysis of focus association with wh-phrases and certain types of focus intervention effects. In sum, to maintain a purely syntactic analysis, one would need to stipulate lexical ambiguity of $d o u$ or switch to a less constrained theory of focus sensitivity in which focus-sensitive operators can (sometimes) use ordinary as well as alternative dimensions the quantification domain. ${ }^{8}$

In comparison, the analysis of this paper assumes a single lexical entry for $\mathrm{dou}$ and a more constrained interpretive mechanism of focus-sensitive operators.

There are, however, two major limitations of the current analysis. First, it does not cover some other dou constructions, e.g., the distributive uses (20). When dou is associated with a definite plural subject (20a), the resulting sentence lacks a collective reading, whereas the same sentence without $d o u$ allows for both collective and distributive readings (20b).

a. Yuehan he Mali dou chi le yi ge pingguo

John and Mary DOU eat PFV one CL apple

'John and Mary each ate an apple.'

collective* $^{*}$ distributive ${ }^{\checkmark}$

b. Yuehan he Mali chi le yi ge pingguo

John and Mary eat PFV one CL apple

'John and Mary ate an apple.'

collective $^{\checkmark}$, distributive ${ }^{\checkmark}$

Second, the two propositional-level operators $[\forall]$ and EVEN postulated for the universal wh ...dou and scalar lian ... dou constructions may seem arbitrary and stipulative, particularly since their relations with dou are treated as a matter of syntactic licensing.

I think a promising way to address these limitations is to integrate the current analysis with recent works on unifying the universal/distributive and the scalar uses of dou (e.g., Xiang 2016a,b; Liu 2017). What they have in common is that they postulate only one alternative-sensitive operator Op (the exact details vary for different theories) together with construction-specific, fine-grained alternative sets for each type of focus associates $\alpha_{F}$, which allows them to derive effects that are equivalent to $[\forall]$, EVEN, and so on for different constructions.

Integrating such approaches to the current analysis means that we only need to postulate one propositional-level operator Op that is in syntactic agreement with $d o u$. It is easy to show that such an analysis will straightforwardly captures grammatical dou constructions where the focus associate $\alpha$ appears above dou: the structural configuration in this case is $\left[\mathrm{Op}\left[\ldots\left[\alpha_{F} \ldots[\right.\right.\right.$ dou ... $\left.\left.\left.]\right]\right]\right]$. Since $d o u$ does not intervene between Op and $\alpha_{F}$, it has no semantic effect and hence Op and $\alpha_{F}$ will interact and

8 It should also be noted that Li \& Law (2016) do not consider all three constructions of dou and it is not clear whether their approach can be extended to account for all three constructions. 
yield the the desired reading according to whichever analysis above that is imported. The more challenging task is to make sure that the other configuration [Op [... [dou $\left.\left.\left.\ldots\left[\alpha_{F} \ldots\right]\right]\right]\right]$ is ungrammatical. This crucially depends on how the alternative values of $\alpha_{F}$ are defined and how Op uses them, which varies for different theories. I will leave the exploration of such possibilities for future work.

Finally, it should be noted that any purely semantic analysis that postulates only a propositional-level operator Op as the meaning of dou (i.e., dou is assumed to always takes wide scope over the entire proposition) is unlikely to capture the full pattern of ordering restrictions. The main challenge comes from exhaustive dou wh-questions. If dou always take wide scope, then it would always scope above the wh-phrase whether it is the subject or object, and we would predict no asymmetry, contrary to fact.

\subsection{Diversity of focus intervention effects}

I have argued that the patterns of $d o u$ constructions can be understood as intervention effects, with the critical configuration being $\left[\mathrm{Op}\left[\ldots\left[\right.\right.\right.$ dou $\left.\left.\left.\ldots\left[\alpha_{F} \ldots\right]\right]\right]\right]$. The literature on focus intervention effects has traditionally centered on cases where Op is the interrogative force and focus intervention leads to ungrammaticality (e.g., Beck 2006; Beck \& Kim 2006). A notable exception is Li \& Law's (2016) observation that an intervening focus-sensitive operator does not necessarily result in ungrammaticality. This paper provides further empirical evidence for this view (although the proposed analysis is different from Li \& Law's: see the discussion in the previous subsection) and suggest a wider range of focus intervention effects than previously considered that are worth further exploration.

\section{Conclusion}

In this paper, I introduce three Mandarin Chinese dou constructions, which have different meanings involving questions and focus sensitivity and are subject to ordering restrictions. I suggest that the seemingly complex patterns can be viewed as intervention effects. Formalizing this idea, I propose a novel and principled way to combine question and focus semantics, and provide a semantics of dou such that the ordering restrictions follow from its interaction with other independently motivated semantic constraints. The current analysis is the first semantic account of the ordering restrictions in dou constructions, and if it is on the right track, would suggest a wider range of focus intervention effects than previously considered that are worth further exploration. 
Mandarin dou constructions

\section{References}

Beck, Sigrid. 2006. Intervention effects follow from focus interpretation. Natural Language Semantics 14(1). 1-56. doi:10.1007/s11050-005-4532-y.

Beck, Sigrid \& Shin-Sook Kim. 2006. Intervention effects in alternative questions. The Journal of Comparative Germanic Linguistics 9(3). 165-208. doi:10.1007/s10828-006-9005-2.

Charlow, Simon. 2014. On the semantics of exceptional scope. New York, NY: New York University dissertation.

Cheng, Lisa Lai-Shen. 1995. On dou-quantification. Journal of East Asian Linguistics 4(3). 197-234. doi:10.1007/BF01731509.

Ciardelli, Ivano, Floris Roelofsen \& Nadine Theiler. 2017. Composing alternatives. Linguistics and Philosophy 40(1). 1-36. doi:10.1007/s10988-016-9195-2.

Giannakidou, Anastasia \& Lisa Lai-Shen Cheng. 2006. (In)definiteness, polarity, and the role of wh-morphology in free choice. Journal of Semantics 23(2). 135-183. doi:10.1093/jos/ffl001.

Hamblin, Charles Leonard. 1973. Questions in Montague English. Foundations of Language 10(1). 41-53. doi:10.2307/25000703.

Kratzer, Angelika \& Junko Shimoyama. 2002. Indeterminate pronouns: The view from Japanese. In Yukio Otsu (ed.), Tokyo conference on psycholinguistics 3, 1-25. Tokyo, Japan: Hituzi Syobo Publishing Company.

Li, Haoze \& Jess H.-K. Law. 2016. Alternatives in different dimensions: a case study of focus intervention. Linguistics and Philosophy 39(3). 201-245. doi:10.1007/s10988-016-9189-0.

Liao, Hsiu-Chen. 2011. Alternatives and exhaustification: Non-interrogative uses of Chinese wh-words. Cambridge, MA: Harvard University dissertation.

Lin, Jo-wang. 1996. Polarity licensing and wh-phrase quantification in Chinese. Amherst, MA: University of Massachusetts, Amherst dissertation.

Lin, Jo-wang. 1997. On wh ... dou constructions. The Tsing Hua Journal of Chinese Studies 27(1). 51-81.

Liu, Mingming. 2017. Varieties of alternatives: Mandarin focus particles. Linguistics and Philosophy 40(1). 61-95. doi:10.1007/s10988-016-9199-y.

Rawlins, Kyle. 2013. (Un)conditionals. Natural Language Semantics 21(2). 111178. doi:10.1007/s11050-012-9087-0.

Roelofsen, Floris \& Donka F. Farkas. 2015. Polarity particle responses as a window onto the interpretation of questions and assertions. Language 91(2). 359-414. doi:10.1353/lan.2015.0017.

Rooth, Mats. 1985. Association with focus. Amherst, MA: University of Massachusetts at Amherst dissertation.

Rooth, Mats. 1992. A theory of focus interpretation. Natural Language Semantics 
1(1). 75-116. doi:10.1007/BF02342617.

Shyu, Shu-ing. 1995. The syntax of focus and topic in Mandarin Chinese. Los Angeles, CA: University of Southern California dissertation.

Tsai, Cheng-Yu. 2015. Toward a theory of Mandarin quantification. Cambridge, MA: Harvard University dissertation.

Xiang, Ming. 2008. Plurality, maximality and scalar inferences: A case study of Mandarin dou. Journal of East Asian Linguistics 17(3). 227-245. doi:10.1007/s10831-008-9025-9.

Xiang, Yimei. 2016a. Interpreting questions with non-exhaustive answers. Cambridge, MA: Harvard University dissertation.

Xiang, Yimei. 2016b. Mandarin particle dou: A pre-exhaustification exhaustifier. Manuscript.

Ciyang Qing

450 Serra Mall, Building 460

Margaret Jacks Hall

Stanford, CA, 94305-2150

USA

qciyang@stanford.edu 